International Journal of Electronics and Communication Engineering and Technology (IJECET)

Volume 10, Issue 2, March-April 2019, pp. 40-46. Article ID: IJECET_10_02_006

Available online at

http://iaeme.com/Home/issue/IJECET? Volume=10\&Issue=2

ISSN Print: 0976-6464 and ISSN Online: 0976-6472

(C) IAEME Publication

\title{
ANALYSIS OF BRAIN ACTIVITY FOR THE COMMUNICATION IN LOCKED IN PEOPLE
}

\author{
Anand I U, Arya Murali, Sneha M Suryakumar, V. Suharsha and Sayyid Abdul Basith \\ Assistant Professor, Department of Electronics and Communication, \\ Malabar College of Engineering and Technology, Thrissur, India
}

\begin{abstract}
Presently, due to the after effect of the inventions, various techniques are a used for control of home appliances. Particularly, they are particularly designed for the aged people and also for patients. In some cases advanced systems are built for the physically disabled people. The main problem in all current systems is that they are not suitable for all kinds of people and under all conditions. Each system needs a unique design and device, which increases the cost of constructing them and reduction in stability. The main aim of our project is to make a a simple system, which can be used by all types of people in every situation for controlling devices around them.

Brain waves can be used to solve this problem. Here, the brain wave has an important role in controlling devices in the home condition and enable communication among people suffering from pseudo coma. The brain waves are detected by use of Neurosky Mindwave sensor and data is transferred using Bluetooth to the CA system. The CA system is made up of an Arduino and its programmed to control all the devices connected. The main advantage of the project is that it can be used by all types of people.
\end{abstract}

Keywords: NeuroskyMindwave, Bluetooth, Central automation system, Pseudocoma.

Cite this Article: Anand I U, Arya Murali, Sneha M Suryakumar, V. Suharsha, Sayyid Abdul Basith, Analysis of Brain Activity for the Communication in Locked in People, International Journal of Electronics and Communication Engineering and Technology, 10(2), 2019, pp. 01-07.

http://iaeme.com/Home/issue/IJECET?Volume=10\&Issue=2

\section{INTRODUCTION}

Locked-in syndrome

Locked-in syndrome (LIS), also known as pseudocoma, is a stae of human body in which a patient is aware but cannot move or communicate verbally due to complete paralysis of nearly all voluntary muscles in the body except for vertical eye movements and blinking. The individual is conscious and sufficiently intact cognitively to be able to communicate with eye movements. The EEG is normal in locked-in syndrome. 
Total locked-in syndrome, or completely locked-in state (CLIS), is a version of locked-in syndrome wherein the eyes are paralyzed as well. Fred Plum and Jerome Posner coined the term for this disorder in 1966.

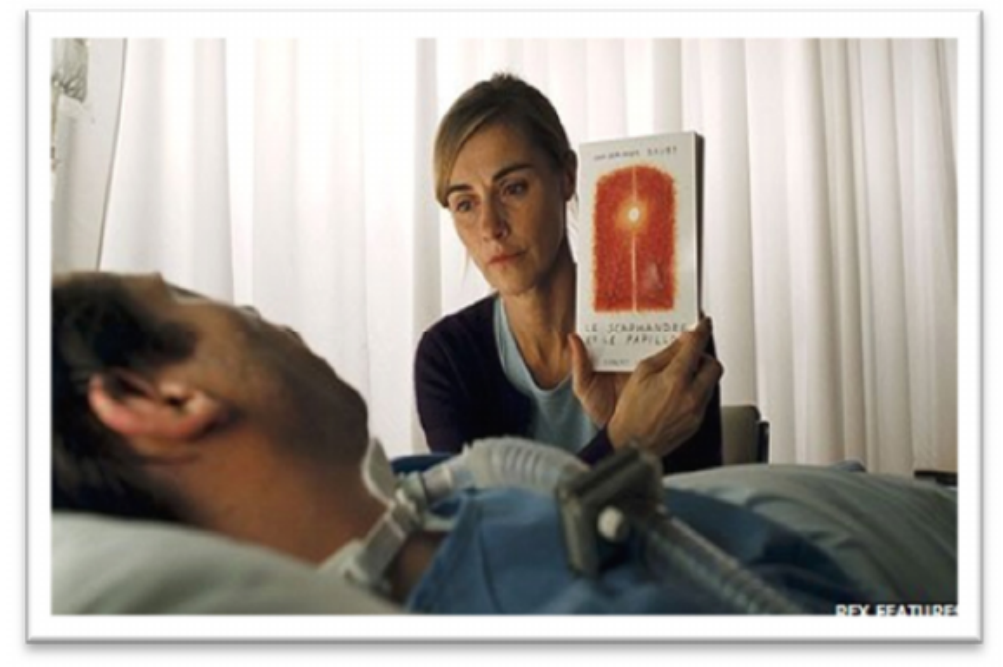

Figure 1 Man suffering from locked-in syndrome

Persons having severe motor disabilities for various reasons can use a wide range of assistive devices (ADs) for managing their daily needs as well as using them for communication and entertainment purposes. The set of ADs ranges from simple switches connected to a remote controller to complex sensors (e.g., mouth mouse) attached to a computer and to eye tracking systems. All of these systems work very well after being adjusted individually for each person. However, there are still situations where the systems do not work properly, e.g., in the case of fatigue of residual muscles. In such a case, a BrainComputer Interface (BCI) might be a good option, using brain signals (EEG) for control without the need for movement.

\section{OBJECTIVES OF THE STUDY}

Two questions were identified to guide the study:

- To analyze the brain activity and thereby help locked in people to communicate and to control the home appliances.

\section{METHODOLOGY}

The first step in the study was to analyze the brain waves of locked in people using Neuroskymindwave headset. A detailed description of the analysis, validation and calibration of the same is discussed below.

\subsection{Neuroskymindwave headset}

\section{Diagram of a basic NeuroSky headset}

The human brain is constructed of billions of interconnected neurons; the regularity of interaction between those neurons are defind as thoughts and emotional conditions. Every communication between neurons creates a minute electrical discharge; to detect these signals outside of the skull is very hard. But the interaction between them results in forming a brain signals or waves which can be detected. 


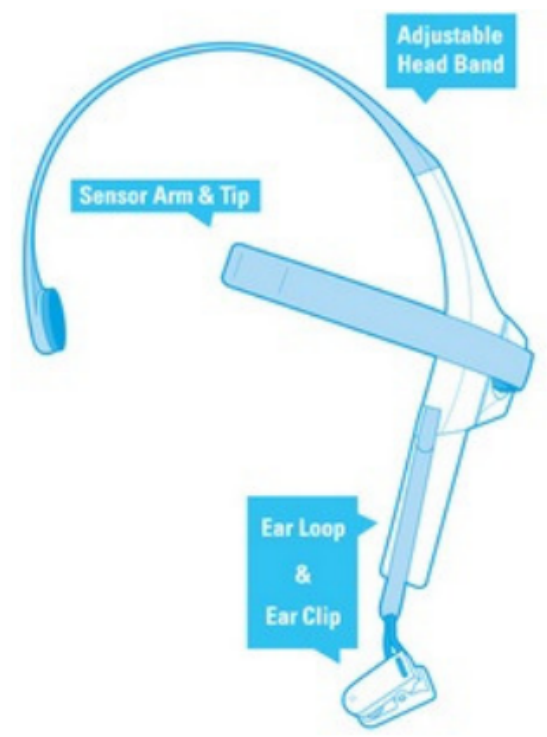

Different brain conditions are due to different patterns or structure of neural interaction. These structure or brain patterns lead to brain waves characterized by different amplitudes and frequencies; for example, brainwaves in between 12 and 30 hertz are classified as Beta Waves, these are linked with concentration; waves between 8 and 12 hertz are c classified as Alpha Waves, these are linked with relaxation and a state of mental calm. (as the muscle expand and close some patterns are generated the sensor detects these for moree accurate data.)

All types of electrical actions produce these signals (including light bulbs), thus all electrical equipments produce some amount of "noise"; this "noise" interact with the waves originating from the brain, that's why most of the EEG devices will pick up readings even when they are not connected to a persons head. Analysing mental activity through these signals is like trying to overhear an conversation at a loud music contest. In the past, EEG devices avoided this problem by analysing these signals in surroundings where electrical activity is rigorously restrained and increasing the signal power of the data coming from the brain through various techniques.

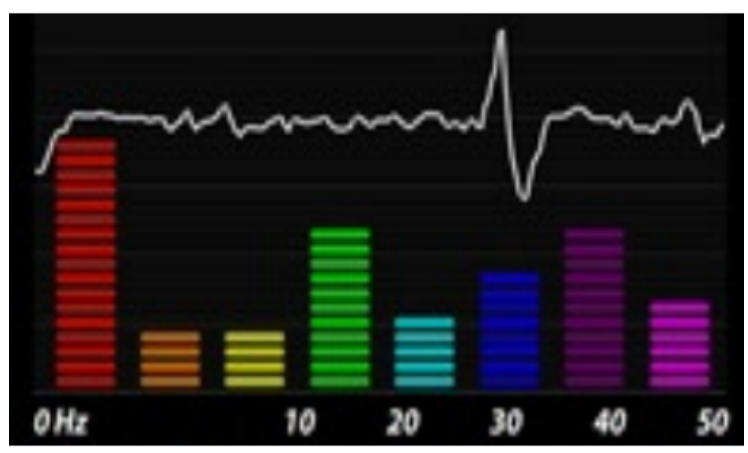

\section{Raw Brainwaves and Power Spectrum}

However, most patients do not have rooms in their house lacking electronic devices nor do the people want to apply a conductive liquid to their head each time they use a BCI device. NeuroSky has found complex algorithms and built it into the products which cleans out this "noise". NeuroSky's white paper claims the Think Gear technology has been tested at $96 \%$ as accurate as that within research grade EEGs. 


\section{How it works}

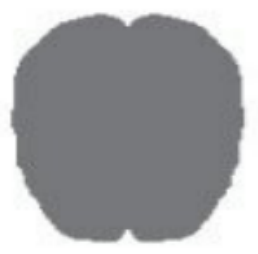

Your Brain

Produces tiny electrical impulses called brainwaves

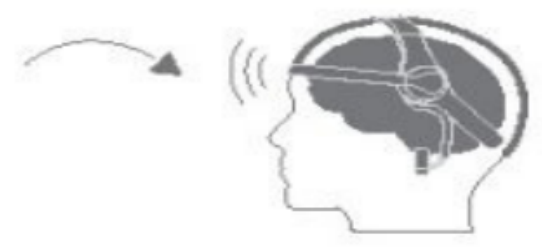

Neuro Headset

Detects these waves and wirelessly communicates

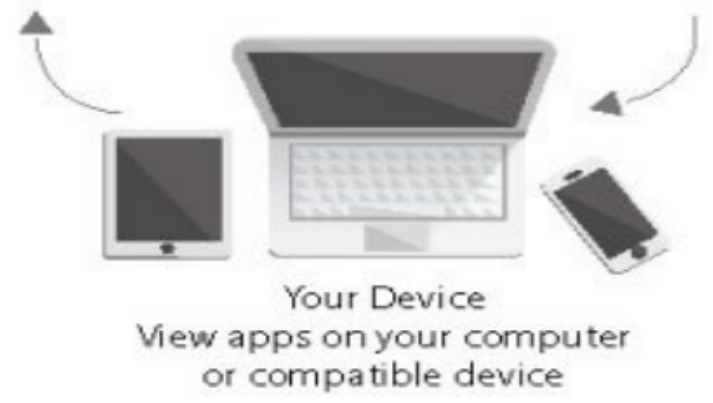

\subsection{Microcontroller}

The ATmega328 is a single-chip microcontroller created by Atmel in the mega AVR family. It has a modified Harvard architecture 8 bit reduced instruction set computer processor core.

The chipis a 8-bit AVR RISC-based microcontroller which consists of $32 \mathrm{kB}$ In System Programming flash memory with read while write capability, 1 kB EEPROM, 2 KB SRAM, 23 general purpose I/O lines, 32 general purpose working registers, 3 flexible timer/counters with compare modes, internal and external interrupts, serial programmable USART, a byteoriented 2-wire serial interface, SPI serial port, 6-channel 10-bit A/D converter, programmable watchdog timer with internal oscillator, and five software selectable power saving modes. The device operates between 1.8-5.5 volts. The device achieves throughput approaching 1 MIPS per MHz.

ATmega 328 consists of a 1KB Electrically Erasable Programmable Read Only Memory (EEPROM). This shows that if the current supply supplied to the micro-controller is terminated, even then it can store the data and can provide results after providing it with the electric supply. Moreover, ATmega-328 has 2KB Static Random Access Memory. ATmega 328 has different features which make it the most popular device in current market. These properties consist of advanced RISC architecture, good performance, low power consumption, real timer counter having separate oscillator, 6 PWM pins, programmable Serial USART, programming lock for software security, throughput up to 20 MIPS etc. 


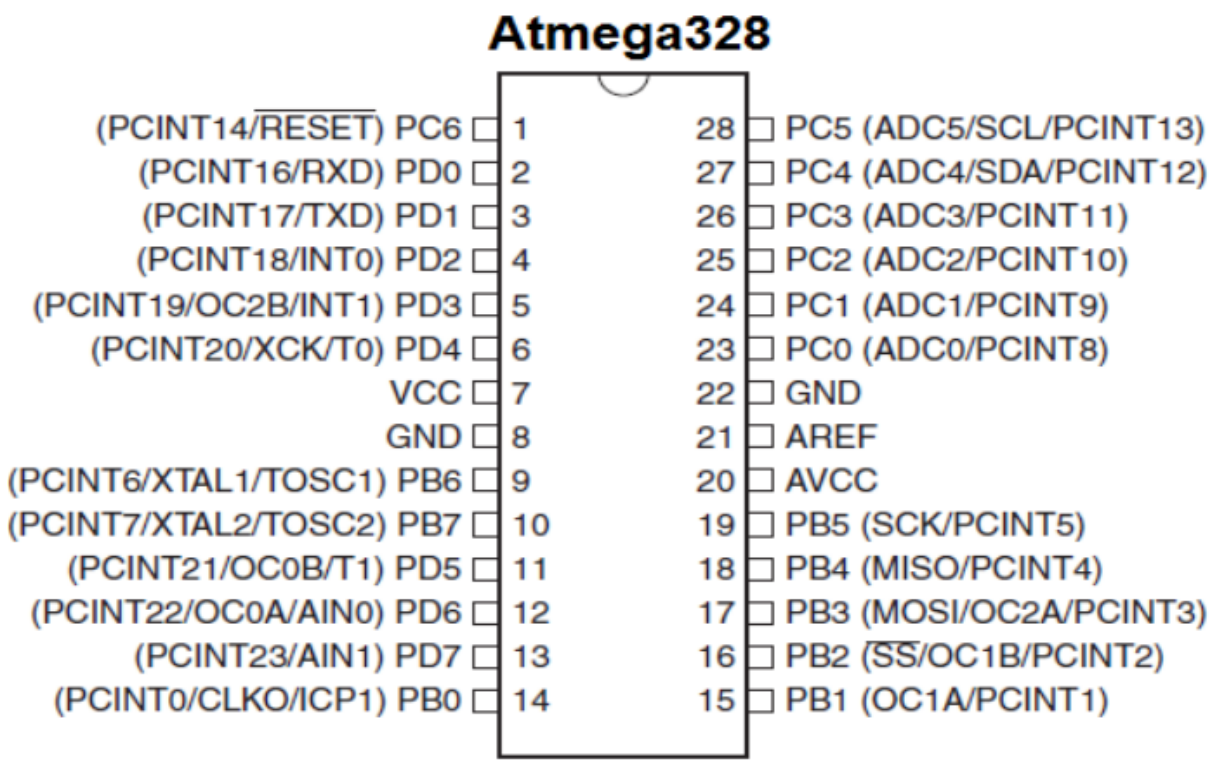

\subsection{HC-05 Serial Bluetooth Module}

Bluetooth is a technology used for wireless communication. It's created to replace cable connections. It make use of serial communication to communicate with devices. It communicates with microcontroller using serial port (USART). Usually, it connects small devices like mobile phones, PDAs and TVs using a short-range wireless connection to exchange documents. It uses the $2.45 \mathrm{GHz}$ frequency band. The connection can be point-topoint or multi-point where the maximum range is 10 meters. The transfer rate of the data is 1 Mbps.

HC-05 Bluetooth module provides switching mode between master and slave mode which means it able to use neither receiving nor transmitting data.

Comparing it to the HC-06 module, which can only be set as a Slave, the HC-05 can be set as Master as well which enables making a communication between two separate Arduino boards.

A Bluetooth module can simply be used for a serial port replacement to establish connection between MCU, PC to the embedded projects and etc.

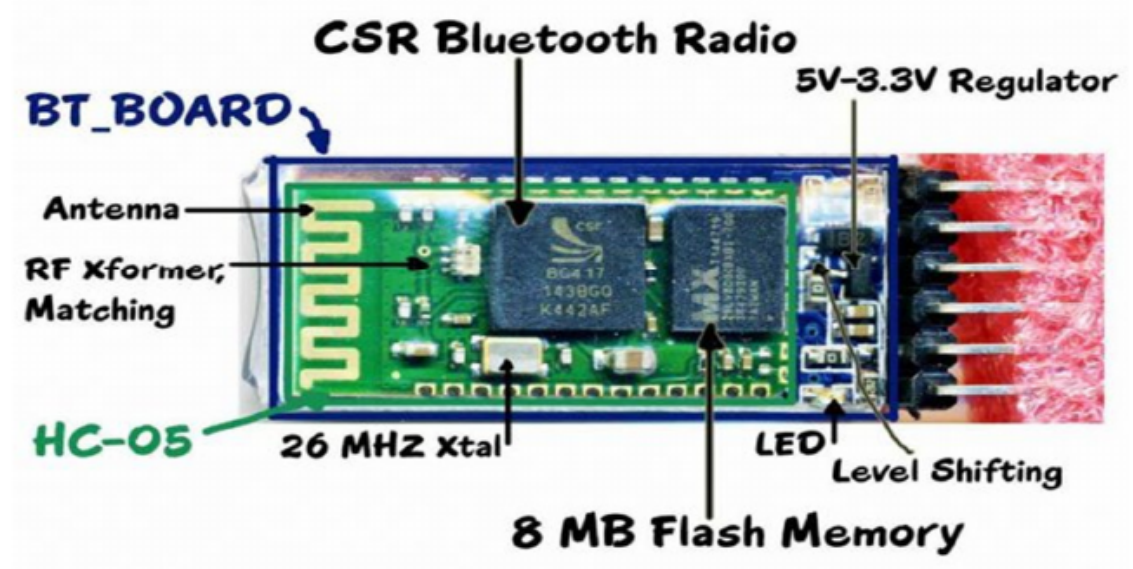




\subsection{APR9301 Voice Recorder}

APR 9301 is a single chip Voice recorder and Playback equipment used for 20 to 30 seconds voice recording and play back. It's an excellent IC for automatic answering machine, door phones etc. This IC has data memorizing capacity and needs no software or microcontroller. It gives high quality voice recording and play back up to 30 seconds.

This ic operates in 2 modes of operation-recording and playback modes. The ic requires only low voltage to function (5 volts).

\section{BLOCK DIAGRAM AND RESULTS}

The block diagram provides with explanation of how the system works. The BCI is highly evolving technique; it can be utilised for many things like controlling appliances, gaming etc. we here define the use of BCI with an APR module for language interpretation and epuipment control

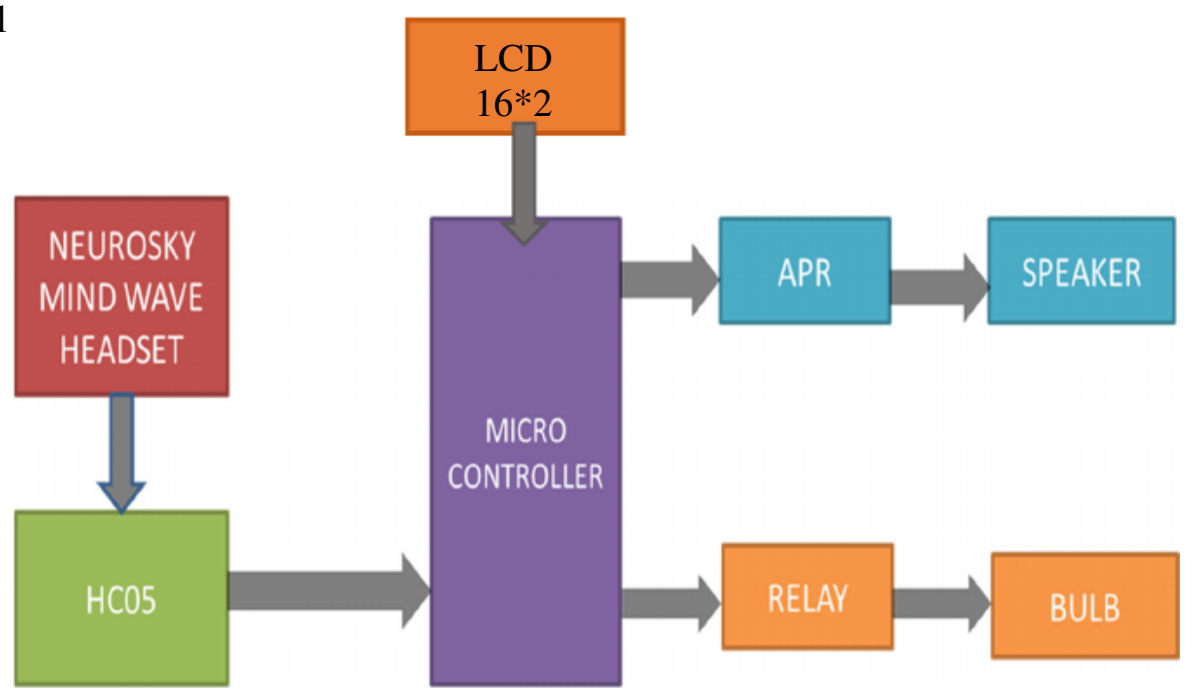

Brainwaves are tiny electrical impulses produced when a neuron fires in the brain. NeuroSky's brain -computer interface (BCI) technology works by monitoring these electrical impulses with a forehead sensor. The brain signals are input into the Think Gear chip, and understood via the patterned Attention and Meditation algorithms. The analysed electrical signals and calculated readings are then provided as output and as digital messages to the computer, toy, or mobile device, allowing you to see your brain waves on the screen, or use your brainwaves to affect the device's behaviour.

HC05 bluetooth module is used here for receiving the datas collected by the Neuroskymindwave headset.HC05 then serially transfers these collected informations to the Atmega328 microcontroller in the aurdino board. The collected datas includes the various mind states such as meditation, concentration and attenuation. A comparison between these collected and saved datas is processed inside the microcontroller and finally provides the required results. The result can be a recorded APR audio, a buzzer, or home automation.

\section{CONCLUSION}

Based on the outcome of the study it is proved that it is no longer required for the locked in people to completely depend on their bystander. They can make use of these simple profile to communicate and in home automation. We can make use of advanced technologies in the proposed system by use of mind reading and mind controlling mechanisms. Further research is required to replicate and clarify these results. 
Anand I U, Arya Murali, Sneha M Suryakumar, V. Suharsha, Sayyid Abdul Basith

\section{REFERENCES}

[1] Anupama.H.S, N.K.Cauvery, Lingaraju.G.M, "Brain Computer Interface and its Types AStudy" International Journal of Advances in Engineering \& Technology, pp. 739-745, 2012.

[2] Haider Hussein Alwasiti, IshakAris and AdznanJantan, "Brain Computer Interface Design and Applications: Challenges and Future", World Applied Sciences Journal 11, pp. 819$825,2010$.

[3] AndreFerreira,Wanderley C Celeste, Fernando A Cheein, Teodiano F Bastos Filho, MaraiSarcinelli Filho and Ricardo Carelli, "Humanmachine interfaces based on EMG and EEG applied to robotic systems", Journal of Neuro Engineering and rehabilitation 2008, 5:10 doi:10.1186/1743-0003-5-10.

[4] Choi B, Jo S, "A Low-Cost EEG System-Based Hybrid Brain-Computer Interface for Humanoid Robot Navigation and Recognition", PLoS ONE 8(9): e74583. doi:10.1371/journal.pone.0074583, 2013.

[5] Haas, S., Frei, M., Osorio, I., Pasik-Duncan, B. \&Radel, J. "EEG Ocular Artefact Removal Through Armax Model System Identification Using Extended Least Squares", Communications in Information and Systems3: 19-40, 2003.

[6] Lai Wei and Huosheng Hu, "Multi-modality - EMG and Visual Based Hands-Free control of an Control of an Intelligent Wheelchair", ICIRA 2010, Part II, LNAI 6425, pp. 659670, 2010. () Springer-Verlag Berlin Heidelberg 2010.

[7] Laufs, H., Kleinschmidta, A., Beyerlea, A., Egera, E., SalekHaddadib, A., C., P. \& Krakow, K, "EEG-Correlated FMRI of Human Alpha Activity", NeuroImage 19(4): 1463-1476, 2003.

[8] Mahmud, M., Hawellek, D. \&Bertoldo,A, "EEG Based Brain-Machine Interface For Navigation Of Robotic Device", Proceedings of the 3rd IEEE/RAS-EMBS International Conference on Biomedical Robotics and Biomechatronics (BioRob2010), Tokyo, Japan, pp. 168-172, 2010. 